\title{
Environmental dependence of inbreeding depression in cultured Coho salmon (Oncorhynchus kisutch): aggressiveness, dominance and intraspecific competition
}

\author{
JA Gallardo and R Neira \\ Laboratorio de Genética Cuantitativa, Departamento de Producción Animal, Facultad de Ciencias Agronómicas, Universidad de Chile, \\ Santa Rosa 11315, La Pintana, Santiago, Chile
}

\begin{abstract}
We evaluated the effects of inbreeding on traits related to territorial dominance and tested whether the magnitude of inbreeding depression (ID) was modified by social environment in Coho salmon. Evaluation of behaviour in paired contests between juvenile salmon with different inbreeding (low, $\mathrm{LI}=9.5 \%$; medium, $\mathrm{MI}=29.6 \%$ ), did not show significant differences between their capacities for establishing territorial dominance (mean aggressiveness score, $\mathrm{LI}=20.0 \pm 22 ; \mathrm{MI}=16.7 \pm 23$ or for feeding attempts, $\mathrm{LI}=18.3 \pm 12 ; \overline{\mathrm{MI}}=21.1 \pm 12)$. However, fish with low inbreeding (LI) showed almost twice the aggressive pursuit of fish with medium inbreeding (MI), and had a higher specific growth rate $(S G R)$ in culture $\left(S G R_{M I}=1.83 \pm 0.58\right.$; $\left.\mathrm{SGR}_{\mathrm{LI}}=2.20 \pm 0.67\right)$. Additionally, we found evidence that the magnitude of ID was modified by social environment: (1)
\end{abstract}

Masking: In small groups of fish $(N=20)$, large dominant fish of MI, cultivated with small subordinate fish of $\mathrm{LI}$, showed the same SGR as dominant fish of $\mathrm{LI}$ cultivated with small subordinate fish of MI. (2) Magnifying: A significant effect of ID on juvenile survival was detected only in high-density competitive environments. Thus, the number of lethal equivalents was 2.70 at high-density, and only 0.24 in a low-density environment. Our results show that differences in size associated with territorial dominance may mask deleterious effects of inbreeding under certain conditions, and support the concept that intraspecific competition usually magnifies the deleterious effects of inbreeding.

Heredity (2005) 95, 449-456. doi:10.1038/sj.hdy.6800741; published online 28 September 2005

Keywords: salmon culture; dominance; inbreeding depression; behavioural traits; Chile

\section{Introduction}

The deleterious effects of inbreeding on fitness have been widely investigated in genetics and evolutionary biology (Lynch and Walsh, 1998) and recently in salmon biology and aquaculture (Wang et al, 2001). This phenomenon, known as inbreeding depression (ID), is usually defined as the reduction of the mean phenotype value of a given trait due to inbreeding (Falconer and Mackay, 1996). There is a general consensus that traits linked to fitness undergo greater ID than traits not directly linked with adaptation, such as morphological traits (Falconer and Mackay, 1996; DeRose and Roff, 1999). Theoretical considerations argue that this is a consequence of traits linked to fitness having greater variance in directional dominance than morphological traits (Lynch and Walsh, 1998).

In juvenile salmon, behavioural traits such as aggressiveness and foraging behaviour have been related to territorial dominance and to specific growth rate (SGR) (Damsgård et al, 1997; Nicieza and Metcalfe, 1999; Lahti

Correspondence: $R$ Neira, Laboratorio de Genética Cuantitativa, Departamento de Producción Animal, Facultad de Ciencias Agronómicas, Universidad de Chile, Santa Rosa 11315, La Pintana, Santiago, Chile.

E-mail:rneira@uchile.cl

Received 18 June 2004; accepted 28 June 2005; published online 28 September 2005 et al, 2001). Both characteristics have been associated with fitness (Quinn and Peterson, 1996), and should therefore be strongly influenced by inbreeding. Under natural conditions juvenile salmon are territorial, and defend small portions of the rivers in which they feed by aggressive acts (Holtby et al, 1993; Nicieza and Metcalfe, 1999; Healy and Lonzarich, 2000). This aggressive behaviour produces hierarchies of dominance in which the dominant individuals usually monopolise the best feeding areas (Valdimarsson and Metcalfe, 2001). This allows them to maximise their benefits in terms of net energy gain (Fausch, 1984), although under some conditions of unpredictability of the food supply or of environmental disturbance, this situation may be reversed (Sloman et al, 2001; Vøllestad and Quinn, 2003), revealing a trade-off between aggressiveness and fitness. In any case, the dominance hierarchy generates a high degree of stress in subordinate fish (Ejike and Schreck 1980; Sloman et al, 2000, 2001) producing, among other physiological consequences, a decrease in disease resistance as well as depressed growth (Sloman et al, 2000). In culture conditions, it has been suggested that the high densities employed would prevent the establishment of territories and that intraspecific competition should be low, given that food is always available. Several authors have, however, demonstrated that some dominant fish may defend portions of culture tanks, create areas of low 
density, monopolise the food and grow better than subordinate individuals (Thorpe et al, 1990; Ryer and Olla, 1996). Also, high culture densities generally increase the frequency of encounters between fish, thus increasing aggressive interactions. These usually produce severe body damage, principally on the fins and to a lesser degree on the eyes and operculum (Abbott and Dill, 1985; Turnbull et al, 1998). The prevalence of damage on fins is so high in some cultured populations that it has been used to differentiate cultured fish from wild fish (Craik et al, 1987).

There are several studies that describe the deleterious effects of inbreeding in fish, generally in captive conditions or in association with artificial selection ( $\mathrm{Su}$ et al, 1996; Pante et al, 2001; Gallardo et al, 2004a). Few have determined whether or not this is a consequence of lower competitive ability of inbred individuals. Tiira et al (2003) recently demonstrated that aggressiveness in Salmo salar was positively correlated with genetic diversity, which is an indirect measure of inbreeding (Hedrick and Kalinowski, 2000). Researchers of other taxa have also shown that consanguineous individuals have lower competitive abilities, for example, in obtaining and defending territory (Meagher et al, 2000). Also, it has been demonstrated that very high inbreeding may limit recognition among individuals and alter behavioural patterns in the presence of potential competitors (Nevison et al, 2000).

From another perspective, prediction of the effects of inbreeding in a population has become a highly complex undertaking, since the magnitude of ID usually varies according to the conditions of the biotic and abiotic environment in which it is evaluated (Hauser and Loeschcke, 1996; Dahlgaard and Loeschke, 1997; Bijlsma et al, 1999; Dahlgaard and Hoffmann, 2000). There is a tendency, however, for the magnitude of ID to increase under competitive conditions or environmental stress (Latter and Mulley, 1995; Crnokrak and Roff, 1999; Hedrick and Kalinowski, 2000; Haag et al, 2002). The genetic basis of this phenomenon seems to be the existence of conditionally deleterious alleles (Crow, 1997; Bijlsma et al, 1999), the effects of which are revealed only under restrictive and not under permissive conditions.

In this study, we evaluated the effects of inbreeding on behavioural traits related to territorial dominance in a domesticated Coho salmon (Oncorhynchus kisutch) population. We specifically investigated the consequences of inbreeding on aggressiveness and feeding activity. Since both of these traits have been strongly linked to territorial dominance and to fitness, we expected to encounter a high degree of ID for them. This could be expressed, for example, if inbred individuals tended to be more subordinate than fish that were not inbred. We also studied the effects of the social environment (territorial dominance and intraspecific competition) on the magnitude of the ID on body weight, both under laboratory and aquaculture conditions. The effect of inbreeding is particularly important in salmon aquaculture because it is based on a high degree of artificial selection, which tends to increase the rate of inbreeding.

The study was carried out with cultured fish from a selective breeding program (Gallardo et al, 2004a, see below). Significant ID has been estimated for some traits related to fitness, such as the gonadosomatic index (GSI,
$-5.3 \%$ for each $10 \%$ increase of inbreeding). Additionally, we have some evidence that the effect of ID on body weight varies according to the environment (environmental dependence). Our previous results showed that the magnitude of ID on body weight increased from the alevins $(5-10 \mathrm{~g})$ to the smolt size $(150 \mathrm{~g})$ during a period in which fish from families having different levels of inbreeding were cultivated together in floating cages in the sea. The overall results of our analyses for the 2000 year-class showed decreases of $1.1 \%$ in weight for each $10 \%$ increase in inbreeding at the alevin stage (before families were mixed); body weight of smolts, after 3 months of communally rearing, showed a $3.8 \%$ decrease for each $10 \%$ increase in inbreeding.

\section{Materials and methods}

\section{Study population}

The study was based on fish from one cultured Coho salmon population belonging to the genetic improvement centre maintained by the Institute for Fisheries Development (IFOP) and the University of Chile in Coyhaique (XI Region, Chile). The population was established in $1992(\mathrm{Ne}=61)$, and was managed in a 2year productive cycle. The normal generation period for wild Coho salmon is 3 or 4 years; however, in Chilean cultured stocks, smolts are normally obtained in less than a year due to the optimal water temperature conditions of the lakes, which reduces the reproductive cycle length. This population has been under standard culture conditions for five generations (10 years). Each year class was produced using a hierarchical mating system by means of mating of 30-35 males with approximately 100 females, producing a population of 30-35 families of half siblings (HS) and 100 families of full siblings (FS). Artificial selection was practised for four generations. Characters selected included weight at harvest, using the best linear unbiased prediction (BLUP) of breeding value with the animal model, and early spawning, by means of phenotypic selection. The inbreeding coefficient of each individual was calculated on the basis of five generations of genealogical records of the animals using the PEDIGREE software (Kinghorn and Kinghorn, 1999). Inbreeding was assumed to be zero in the base population; the last generation (year-class 2000) had a mean inbreeding coefficient of $9.4 \pm 2.7 \%$ (Gallardo et al, 2004a). Martinez et al (1999) and Neira et al (2004) have given more details on characters and origins of the populations.

\section{Inbred families}

Coho salmon broodstock from the 2002 year-class were bred to produce six full-sib families, with different levels of inbreeding. Low and high-inbred fishes were produced by artificially mating individuals with low and high levels of coancestry (six different pairs of sires and dams). Therefore, individuals from each family had the same level of inbreeding. Coancestry of parents was calculated using the PEDIGREE program (Kinghorn and Kinghorn, 1999). One group of three families had a low level of inbreeding $(F=9.5 \pm 0.3 \%)$, while a second group of three families had an intermediate level of inbreeding $(F=29.6 \pm 0.9 \%)$. The six families were cultivated in separate tanks, with about 1000 fish per tank for 7 
months, until they reached a mean weight of $7 \mathrm{~g}$. Subsequently, 300 fish from each family were randomly placed in systems for observation as follows.

\section{Behaviour in paired contests and analysis of ID}

Behaviour related to territorial dominance was evaluated with 21 pairs of fish, testing medium inbreeding (MI) vs low inbreeding (LI) as indicated below. Fish from four families of the previously mentioned groups were paired by size and cultivated in independent $30 \times 30 \times 30 \mathrm{~cm}^{3}$ aquaria for 17.5 days $(S D=3.8)$. No systematic initial differences existed in pairs of fish that could bias the outcome of competition, neither for body weight nor for fork length. Average body weight of MI and LI groups were 5.35 and $5.34 \mathrm{~g}$, respectively, so the mean body weight difference per pair of fish was $0.01 \mathrm{~g}$. Average fork length of MI and LI groups were 75.9 and $75.7 \mathrm{~mm}$, respectively, thus the mean fork length difference per pair of fish was $0.2 \mathrm{~mm}$. The fish were fed twice a day with artificial food at $6 \%$ of the initial body weight. At the end of the culture period, body length and weight were determined to obtain the specific growth rate SGR $=100\left(\ln W_{2}-\ln W_{1}\right) t^{-1}$, where $W_{1}$ and $W_{2}$ represent the final and initial weights $(\mathrm{g})$ over the culture period $t$ (days). Each fish in the aquarium was labelled with a subcutaneous unique dye mark which allowed its identification (Kelly, 1967). Other studies have shown that this marking technique has no adverse effects on the fishes, nor on their competitive behaviour (Sloman et al, 2001), which we verified in small-scale pilot testing. A $10 \mathrm{~cm}$ length of PVC tubing, $6 \mathrm{~cm}$ diameter, was placed in each test aquarium to provide a refuge to protect subordinate individuals from chronic stress, and to favour establishment of territories. Generally, it was observed that subordinate fish spent more time within the refuge.

Behaviour was recorded by direct observation and using video recording, beginning one day after placing the fish in the aquaria. Fish were isolated from each other by a solid barrier, which prevented visual contact between them; the barrier was removed just prior to the first behavioural observation in each aquarium. Various types of aggressive and feeding behaviours have previously been described in salmonids (Metcalfe et al, 1989; Sloman et al, 2000, 2001; Griffiths and Armstrong, 2002). Table 1 lists the different aggressive behaviours

Table 1 Scoring system used to rate aggressiveness interaction in paired Coho salmon juveniles, one with LI and the other with an MI

\begin{tabular}{lc}
\hline Behaviour & $\begin{array}{c}\text { Score in each } \\
\text { observation }\end{array}$ \\
\hline $\begin{array}{l}\text { Agonistic interactions } \\
\text { Biting another fish }\end{array}$ & 4 \\
Chasing another fish (with or & 3 \\
without biting) & 2 \\
Charging another fish & 1 \\
Lateral display & 0 \\
Receiving or avoiding an & \\
aggressive behaviour of another &
\end{tabular}

When the two fish showed the same behavior, each received the same score, and it was assumed that the higher the score, the higher the degree of dominance. presently evaluated, with the point weight assigned to each of these (1-4) following dominance behaviours: lateral display $<$ Charge $<$ Chase $<$ Biting. An aggressiveness score was calculated for each fish, assigning point values for each behaviour displayed by the aggressor. A totally subordinate fish was scored as zero. Thus if one fish attacked another with a bite, and received a charge in return, the first fish scored four points while the second only two points. On the other hand, if the two fish demonstrated the same behaviour at the same time, each fish received the same score. The amount of food consumed was employed as an additional measure of dominance, observing the number of pellets consumed by each fish during each observational period. For this, feeding times were made to coincide with observational periods, usually once in the morning and once in the afternoon. The feeding behaviour was termed 'attempt to feed' since video recordings and direct observations failed to provide an exact record of how many pellets were ingested. Dominance behaviour was observed 1-2 times a day for 2 days, for 25-30 min periods each. All observations were made from behind a darkened screen located at $1 \mathrm{~m}$ distance from the aquaria so as not to interfere with the behaviour of the fish.

An analysis of principal components (PCA) was carried out to determine which fish of each pair was dominant, using the total aggressiveness score and the mean value of the score obtained on 'attempt to feed'. The fish with the highest factor score within each pair was termed 'dominant' if the difference in dominance scores between the pair was greater than one.

An analysis of variance (ANOVA) was used to detect the effects of inbreeding on aggressiveness and feeding, using the GLM procedure (SAS, Statistical Analysis Systems Institute Inc., 1996). The analysis was carried out using a mixed linear model, which included inbreeding level as a fixed effect, and family within inbreeding as a random effect. Since the aggressiveness score deviated slightly from normality, the analysis was carried out after applying a square root transformation to the variable. Also, an analysis of covariance (ANCOVA) was used to estimate the effect of dominance and inbreeding on the SGR. As above, a mixed linear model was used which included the dominance score as the covariate.

Social environment and ID

Territorial dominance: Body size also has a strong effect on whether one fish will be dominant over another in competitive interactions between juvenile salmonids (Huntingford et al, 1990; Holtby et al, 1993). Generally, larger fish tend to be dominant over the smaller ones. The effect of social environment (territorial dominance) on ID was determined by modifying the body size relation among inbred and noninbred fish. Two groups of 10 fish each were established by body size from the four Coho families described above, with one group consisting of large, dominant individuals, and the other of small, subordinate individuals (Table 2). The group of large fish with LI was cultured with the group of small fish with a medium level of inbreeding. A parallel experiment was carried out with this arrangement reversed. Weights and lengths of all fish were obtained 
after 14 days in culture, and the condition factor, SGR for a given body size, and magnitudes of ID were determined. The differences between members of the same size group but with different levels of inbreeding were analysed for each pair of families using a $t$-test. In this way, we were able to compare the performance of the more inbred fish under two different environmental conditions: dominance and subordination.

Competitive conditions in sea cages: In order to evaluate the magnitude of ID under culture conditions, a group of 60 fish randomly drawn from each family were individually marked using passive integrated transponder (PIT) tags, and then individually measured for weight and length. These fish were intermixed in January 2002 and placed in Ensenada Baja Bay (XI Region, Chile) in floating cages measuring $5 \times 5 \times 4 \mathrm{~m}^{3}$ $\left(100 \mathrm{~m}^{3}\right)$ to give $N=180$ fish per cage and 30 fish per family. Each cage contained an additional different number of fish, which allowed evaluation of the effect of density on the magnitude of ID. We did not measure the degree of aggressiveness in sea culture conditions; however, all fish had the same origin, the genetic improvement centre. The initial number of fish in cages 1 and 2 were 2020 and 3738, respectively, producing an initial density of 20 fish per $\mathrm{m}^{3}$ in cage 1 and 38 fish per $\mathrm{m}^{3}$ in cage 2 . Both groups were fed with pellets using standard culture procedure. After 78 days in culture the body weights, SGR, and survival were measured for all the fish in each group and environment.

An ANOVA was carried out (GLM procedure, see above) to determine the effects of inbreeding, environment and interactions on body weight and SGR. For this we used a mixed linear model, which included inbreeding and density as fixed effects, and family within inbreeding as the random effect. The effect of inbreeding on survival was evaluated using a t-test following

Table 2 Body weight (g), fork length ( $\mathrm{mm})$, and condition factor for larger and smaller juvenile Coho salmon with low $(F$ mean $=10 \%)$ and medium ( $F$ mean $=29.5 \%$ ) levels of inbreeding per couple of family reared in the same aquarium

\begin{tabular}{|c|c|c|c|c|c|}
\hline \multirow[t]{2}{*}{ Trait } & \multirow[t]{2}{*}{ Family } & \multirow[t]{2}{*}{ F } & \multicolumn{2}{|c|}{ Size group } & \multirow[t]{2}{*}{$\mathrm{N}$} \\
\hline & & & Larger & Smaller & \\
\hline \multirow[t]{4}{*}{ BW (g) } & 204 & Medium & $6.66 \pm 0.16$ & $4.78 \pm 0.16$ & 20 \\
\hline & 198 & Low & $6.79 \pm 0.12$ & $4.82 \pm 0.09$ & 20 \\
\hline & 196 & Medium & $6.45 \pm 0.12$ & $4.59 \pm 0.08$ & 18 \\
\hline & 208 & Low & $6.37 \pm 0.06$ & $4.64 \pm 0.07$ & 18 \\
\hline \multirow[t]{4}{*}{$\mathrm{FL}(\mathrm{mm})$} & 204 & Medium & $83.2 \pm 0.5$ & $75.1 \pm 0.8$ & 20 \\
\hline & 198 & Low & $83.7 \pm 0.6$ & $74.4 \pm 0.8$ & 20 \\
\hline & 196 & Medium & $82.10 \pm 0.43$ & $73.10 \pm 0.82$ & 18 \\
\hline & 208 & Low & $83.10 \pm 0.46$ & $74.30 \pm 0.56$ & 18 \\
\hline \multirow[t]{4}{*}{$\mathrm{CF}$} & 204 & Medium & $1.14 \pm 0.02$ & $1.11 \pm 0.03$ & 20 \\
\hline & 198 & Low & $1.14 \pm 0.01$ & $1.15 \pm 0.02$ & 20 \\
\hline & 196 & Medium & $1.15 \pm 0.01$ & $1.16 \pm 0.03$ & 18 \\
\hline & 208 & Low & $1.09 \pm 0.01$ & $1.11 \pm 0.02$ & 18 \\
\hline
\end{tabular}

$\mathrm{BW}=$ body weight; $\mathrm{FL}=$ fork length; $\mathrm{CF}=$ condition factor $=$ $\mathrm{BW} \times \mathrm{FL}^{-3} ; F=$ inbreeding level.

Values \pm standard error. angular transformation of the data. Genetic load was also determined, to allow comparison with other studies, by calculating the number of lethal equivalents (Morton et al, 1956). When there are two groups having different levels of inbreeding, Lynch and Walsh (1998) recommend using the following equation for estimation of the genetic load:

$$
B=-\ln \left(S_{\mathrm{F}} / S_{\mathrm{O}}\right) / F
$$

where $S_{\mathrm{O}}$ and $S_{\mathrm{F}}$ are the probabilities of survival of the groups with LI and $\mathrm{MI}$, respectively, and $F$ is the difference in inbreeding between the groups; $B$ gives an estimation of the reduction in biological adaptation due to inbreeding, and gives the number of lethal equivalents per gamete.

\section{Results}

Analysis of ID in behavioural traits in paired contests A total of 319 aggressive acts (TA) and 2852 'feeding attempts' (FA) were recorded for 21 pairs of fish during $2190 \mathrm{~min}$ of observation (mean $=104 \mathrm{~min}$ per pair; $\mathrm{SD}=18 \mathrm{~min}$ ). Aggressiveness and feeding behavior were highly variable at the individual level for the two levels of inbreeding evaluated (Table 3); however, the individual aggression score was positively correlated with FA $(r=0.55$, Pearson linear correlation coefficient, $P<0.001$; Figure 1).

Inbreeding did not significantly affect the level of aggressiveness $\left(F_{1.38}=0.19 ; P=0.62\right)$, nor did it affect the average number of FA $\left(F_{1.38}=0.43 ; P=0.51\right.$, Table 3$)$.

Table 3 Means and standard deviations (SD) of feeding attempts, aggression score and dominance score (first principal component) by inbreeding level in juvenile Coho salmon

\begin{tabular}{lccccc}
\hline Level of inbreeding & $\mathrm{N}$ & $T A$ & $A S(S D)$ & $T E$ & $F E(S D)$ \\
\hline LI & 21 & 177 & $20.0(22)$ & 1328 & $18.3(12)$ \\
MI & 21 & 142 & $16.7(23)$ & 1524 & $21.1(12)$ \\
\hline
\end{tabular}

$\mathrm{LI}=$ low inbreeding $(F$ mean $=9.5 \%) ; \mathrm{MI}=$ medium inbreeding $(F$ mean $=29.6 \%) ; \mathrm{TA}=$ total agonistic acts; $\mathrm{AS}=$ average aggressiveness score; $\mathrm{TE}=$ total feeding attempts; $\mathrm{FE}=$ average feeding attempts $(\mathrm{N} / 30 \mathrm{~min})$

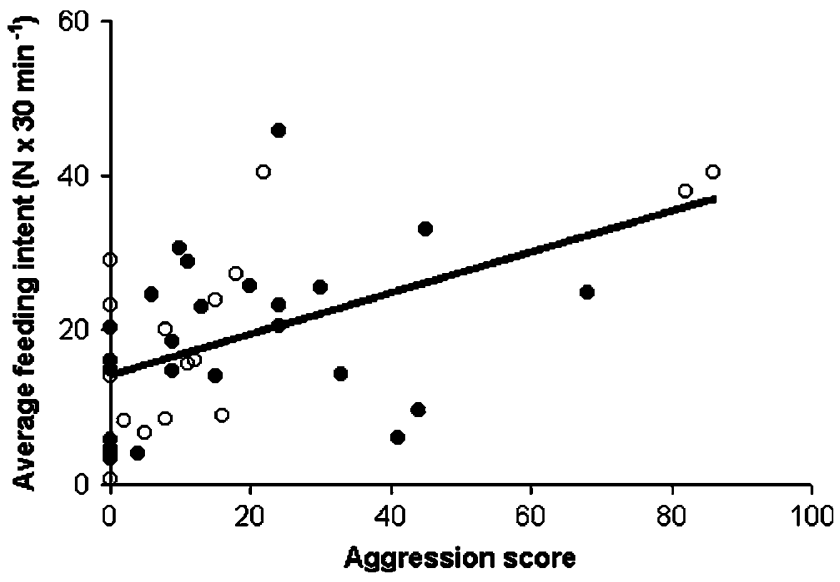

Figure 1 Relationship between aggression score and average FA of low inbred (open circles) and medium inbred (solid circles) Coho salmon in paired contests. 
There was, however, a tendency for fish with a low level of inbreeding to demonstrate almost double the aggressive chases against fish of $\mathrm{MI}$, compared to fish of MI against those of LI (Figure 2). The PCA analysis of the two behavioural variables produced a territorial dominance (factor) score from the first PCA that explained $77 \%$ of the observed variance. Based on this score (range $=5.1$, maximum difference $=3.5$, minimum difference $=0.1$ ) territorial dominance of one fish could be clearly established in 14 pairs while in the remaining seven pairs no dominance relation could be shown (ie difference in score of less than one point). The analysis of the dominance score showed that in eight cases the more inbred fish was dominant, while in only six cases the fish of LI was dominant. By this method, there was no evidence that that inbreeding modified the ability to establish short-term (1-2 day) territorial dominance in fish paired by size. In contrast, the dominance score and the inbreeding significantly affected the specific growth rate (Table 4), with the fish of MI having a lower SGR than those of LI (SGR mean of MI group $=1.83, S D=0.58$; $S G R$ mean of $L I$ group $=2.20$, $\mathrm{SD}=0.67)$.

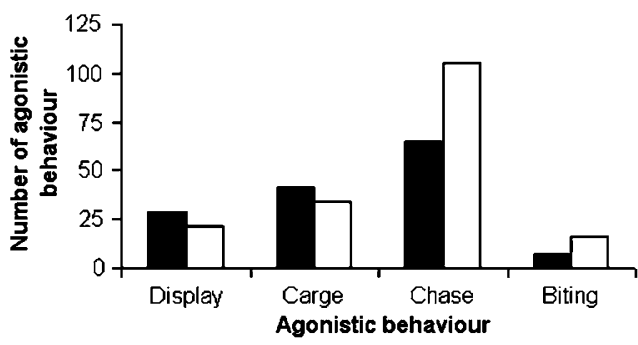

Figure 2 Distribution of agonistic behaviours in paired contests between juvenile Coho salmon with low (open bars) and medium (solid bars) inbreeding.
Analysis of social environment on ID

Territorial dominance: masking effect: Table 5 shows the effects of territorial dominance on ID in two independent experiments (families 196 vs 208 and 204 vs 198; MI vs LI, respectively). In both experiments, large dominant fish of MI, cultivated with small subordinate fish of LI, showed the same BW, FL, CF and SGR as dominant fish of LI cultivated with small subordinate fish of MI. This suggests that the social dominance status (dominant) masked the deleterious effects of inbreeding. This effect was not consistently shown in small fish, as in only one of our experiments both LI and MI fish had similar morphological values (families 196-208). In the other experiment (families 204-198), small subordinate fish of LI had a greater CF ( $t$-test $=-1.96, P=0.03)$ and a greater SGR than subordinate fish of MI $(t$-test $=-2.58$, $P=0.01)$, revealing an ID of low magnitude $(\mathrm{FC}=0.07$; $S G R=0.14)$. Surprisingly, the mean SGR for the subordinate fish of LI was also greater than the SGR of the large, dominant fish of MI $(t$-test $=2.50, P=0.01)$ sharing the same culture aquarium, and greater than their large, dominant, full-sib of LI ( $t$-test 1.90, $P=0.03$ ). Thus, the dominance status (subordination) did not consistently modify the deleterious effect of inbreeding on the SGR.

Table 4 ANCOVA of the effects of inbreeding, family within inbreeding and dominance score (covariate) on SGR $(\% / \mathrm{g} /$ day) using type III sum of squares

\begin{tabular}{lrcc}
\hline Source of variation & $d f$ & $M S$ & $\mathrm{P}$ \\
\hline Inbreeding & 1 & 1.58 & 0.037 \\
Family within inbreeding & 2 & 0.89 & 0.084 \\
Dominance score (covariate) & 1 & 1.80 & 0.027 \\
Residual & 37 & 0.34 &
\end{tabular}

$\mathrm{df}=$ degrees of freedom; $\mathrm{MS}=$ mean squares; $\mathrm{P}=$ probability.

Data satisfied parametric requirements without transformation.

Table 5 Body weight $(\mathrm{g})$, fork length $(\mathrm{mm})$, condition factor and SGR (\% body weight/day) for larger and smaller juvenile Coho salmon with low $(F$ mean $=9.8)$ and medium $(F$ mean $=29.3)$ level of inbreeding

\begin{tabular}{|c|c|c|c|c|c|c|c|}
\hline \multirow[t]{2}{*}{ Family } & \multirow{2}{*}{$\begin{array}{l}\text { Trait } \\
>\end{array}$} & \multirow[t]{2}{*}{ F } & \multicolumn{2}{|c|}{ Size group } & \multirow[t]{2}{*}{ Trait } & \multicolumn{2}{|c|}{ Size group } \\
\hline & & & Larger & Smaller & & Larger & Smaller \\
\hline \multirow{8}{*}{$\begin{array}{l}196 \\
208\end{array}$} & \multirow{4}{*}{ BW (g) } & Medium & $9.24 \pm 0.27$ & $6.53 \pm 0.12$ & \multirow{4}{*}{$\mathrm{FL}(\mathrm{mm})$} & $89.0 \pm 0.70$ & $79.9 \pm 1.05$ \\
\hline & & Low & $9.04 \pm 0.17$ & $6.65 \pm 0.13$ & & $89.2 \pm 0.39$ & $80.9 \pm 0.59$ \\
\hline & & & $-0 . \overline{0} 2$ & $0 . \overline{0} 2$ & & $0 . \overline{0} 0$ & $0 . \overline{0} 1$ \\
\hline & & $P(t$-test $)$ & 0.28 & 0.25 & & 0.40 & 0.21 \\
\hline & \multirow[t]{4}{*}{$\mathrm{CF}$} & Medium & $1.29 \pm 0.02$ & $1.27 \pm 0.04$ & \multirow[t]{4}{*}{ SGR } & $2.55 \pm 0.11$ & $2.53 \pm 0.10$ \\
\hline & & Low & $1.26 \pm 0.01$ & $1.24 \pm 0.01$ & & $2.50 \pm 0.10$ & $2.57 \pm 0.09$ \\
\hline & & & $-0 . \overline{0} 2$ & $-0 . \overline{0} 2$ & & $-0 . \overline{0} 2$ & $0 . \overline{0} 2$ \\
\hline & & $P(t$-test $)$ & 0.08 & 0.23 & & 0.36 & 0.37 \\
\hline \multirow{8}{*}{$\begin{array}{l}204 \\
198\end{array}$} & \multirow[t]{4}{*}{ BW (g) } & Medium & $9.66 \pm 0.27$ & $6.88 \pm 0.25$ & \multirow[t]{4}{*}{$\mathrm{FL}(\mathrm{mm})$} & $91.11 \pm 0.56$ & $81.89 \pm 0.84$ \\
\hline & & Low & $9.87 \pm 0.17$ & $7.40 \pm 0.12$ & & $91.00 \pm 0.47$ & $82.22 \pm 1.09$ \\
\hline & & & $0 . \overline{0} 2$ & $0 . \overline{0} 7$ & & $-0 . \overline{0} 0$ & $0 . \overline{0} 0$ \\
\hline & & $P(t$-test $)$ & 0.26 & 0.06 & & 0.44 & 0.41 \\
\hline & \multirow[t]{4}{*}{$\mathrm{CF}$} & Medium & $1.26 \pm 0.03$ & $1.23 \pm 0.02$ & \multirow[t]{4}{*}{ SGR } & $2.65 \pm 0.10$ & $2.60 \pm 0.13$ \\
\hline & & Low & $1.29 \pm 0.01$ & $1.32 \pm 0.02$ & & $2.73 \pm 0.11$ & $3.05 \pm 0.12$ \\
\hline & & & $0 . \overline{0} 2$ & 0.07 & & $0 . \overline{03}$ & $0 . \overline{14}$ \\
\hline & & $P(t$-test $)$ & 0.14 & 0.03 & & 0.30 & 0.01 \\
\hline
\end{tabular}

$\mathrm{BW}=$ body weight; $\mathrm{FL}=$ fork length $\mathrm{CF}=$ condition factor $=\mathrm{BW} \times \mathrm{FL}^{-3} ; F=$ inbreeding level; $\mathrm{SGR}=$ specific growth rate; $\mathrm{ID}=$ inbreeding depression measured as $\delta=1-\left(X_{\mathrm{MI}} / X_{\mathrm{LI}}\right)$, where $X_{\mathrm{MI}} y X_{\mathrm{LI}}$ is the average of each trait in the group with MI and LI, respectively.

Values \pm standard error. 
Competitive condition: magnifying effect: Inbreeding slightly but significantly reduced the body weight of juvenile salmon (alevin stage) when fish of LI and MI were cultivated in separate aquaria (Table 6). This same group of fish demonstrated significant ID of greater magnitude when cultured under competitive conditions

Table 6 ID for body weight (g) and fork length ( $\mathrm{mm}$ ). Each group is a random sample of three families ( $N=60$ fish per family) reared in independent tanks

\begin{tabular}{lccc}
\hline Mean inbreeding $(S D)$ & $\mathrm{N}$ & Fork length $(S D)$ & Body weight $(S D)$ \\
\hline $9.5(0.3)$ & 180 & $78.1(4.0)$ & $5.60(0.95)$ \\
$29.6(0.9)$ & 180 & $75.1(6.3)$ & $5.09(1.30)$ \\
ID & & $0.04^{*}$ & $0.09^{*}$ \\
\hline
\end{tabular}

ID, ID measured as $\delta=1-\left(X_{\mathrm{MI}} / X_{\mathrm{LI}}\right)$, where $X_{\mathrm{MI}} y X_{\mathrm{LI}}$ is the average of each trait in the group with $\mathrm{MI}$ and LI, respectively. ${ }^{*}=P<0.05$.

Table 7 ID for body weight (g) and for SGR (\%/day) on juvenile Coho salmon reared in two initial density conditions

\begin{tabular}{lcccc}
\hline $\begin{array}{l}\text { Initial density } \\
\left(\text { fish } \times m^{-3}\right)\end{array}$ & $\begin{array}{c}\text { Mean } \\
\text { inbreeding (SD) }\end{array}$ & $\mathrm{N}$ & $\begin{array}{c}\text { Body } \\
\text { weight }\end{array}$ & SGR \\
\hline 20 & $9.4(0.9)$ & 65 & $92.71 \pm 6.13$ & $3.36 \pm 0.11$ \\
& $29.6(0.3)$ & 62 & $66.25 \pm 6.17$ & $3.01 \pm 0.12$ \\
& ID & & $0.29^{* * *}$ & $0.10^{* * *}$ \\
38 & & & & \\
& $9.4(0.9)$ & 62 & $94.82 \pm 7.20$ & $3.38 \pm 0.12$ \\
& $29.6(0.3)$ & 36 & $63.47 \pm 8.69$ & $2.89 \pm 0.11$ \\
& ID & & $0.33^{* * *}$ & $0.14^{* * *}$ \\
\hline
\end{tabular}

$\mathrm{ID}=$ inbreeding depression measured as $\delta=1-\left(X_{\mathrm{MI}} / \mathrm{X}_{\mathrm{LI}}\right)$, where $X_{\mathrm{MI}} y X_{\mathrm{LI}}$ is the average of each trait in the group with MI and LI, respectively. ${ }^{* * *} P<0.001$.

Values \pm one standard error.

Table 8 ANOVA of the effects of inbreeding, family within inbreeding, density and their interactions on smolt body weight and SGR $(\% / g /$ day) using type III sum of squares

\begin{tabular}{lccccccc}
\hline Source of variation & \multicolumn{3}{c}{ Body weight } & & \multicolumn{2}{c}{$S G R$} \\
\cline { 2 - 4 } & $d f$ & F-value & P-value & & F-value & P-value \\
\hline Inbreeding & 1 & 26.48 & $<0.0001$ & & 22.14 & $<0.0001$ \\
Family (inbreeding) & 4 & 24.93 & $<0.0001$ & & 27.15 & $<0.0001$ \\
Density & 1 & 0.08 & 0.775 & & 0.34 & 0.558 \\
Inbreeding $\times$ density & 1 & 0.46 & 0.496 & & 0.70 & 0.405
\end{tabular}

$\mathrm{df}=$ degrees of freedom; $\mathrm{MS}=$ mean squares; $\mathrm{P}=$ probability.

Data satisfied parametric requirements without transformation.

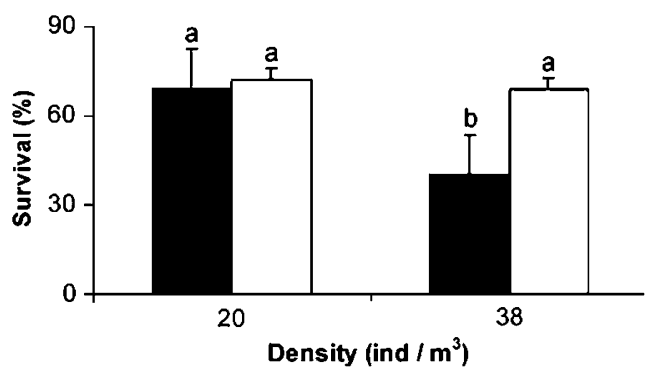

Figure 3 ID on survival (mean SD from three families) of salmon reared from alevin to smolt, as function of density (one sea cage per density). Open and solid bars indicate low and MI, respectively. between families from the alevin to the smolt stages (Table 7). In this experiment, there was a tendency for an increase in the magnitude of ID on body weight and SGR with increase in the culture density. No significant interaction between inbreeding and density was detected for these traits, however (Table 8). On the contrary, survival from alevin to smolt was significantly reduced by inbreeding $(t$-test $=3.76, P<0.01)$, but only in the high-density group, suggesting a strong interaction between inbreeding and density on viability (Figure 3 ). Thus, the number of lethal equivalents expressed in the higher density culture was 2.70, while in the lower density culture it was only 0.24 .

\section{Discussion}

\section{Inbreeding and behaviour}

This study has shown that inbreeding significantly reduced the growth and viability of juvenile fish under different environmental conditions. Other studies on fish have also concluded that inbreeding significantly reduces this and other traits related to fitness under cultured conditions (Su et al, 1996; Gallardo et al, 2004a).

Through the study of behavioural parameters, we evaluated whether or not the deleterious effect of inbreeding was the consequence of lower competitive ability in more inbred fish. Contrary to our hypothesis, we did not find significant differences in the two components that determine competitive ability (aggressiveness and feeding behaviour) in paired contests between fish with two different levels of inbreeding. At least two previous results indicated that aggressiveness or territorial dominance should have been affected by inbreeding. First, both behavioural characteristics have been strongly associated with fitness in juvenile salmonids, for which they should have expressed a high level of ID (Falconer and Mackay, 1996). Second, previous data on salmonids and other vertebrates have shown comparatively lower levels of aggressiveness (Tiira et al, 2003) and of competitive ability in the defence of territory (Meagher et al, 2000) in more inbred individuals. Our study contrasts with the results of Tiira et al (2003), who recently showed that fry of Salmo salar with low genetic diversity were less aggressive than fry with a high degree of genetic diversity. However, since the behavioural evaluations of Tiira et al (2003) were carried out between individuals with the same degree of genetic diversity, their study did not exclude the possibility that genetically similar fish might be less aggressive towards each other. Although our results do not agree with Tiira et al (2003), there is a clear and common tendency for less consanguineous individuals to express a greater number of highly aggressive behaviours such as chases and biting, which may be determinant in the establishment of territories over the long term (Cut et al, 2002). The latter authors showed that, for Salmo salar in a new environment, the dominant fish are the most aggressive, but consume less food with the objective of maintaining a territory over a long term. This tendency was observed in our results for aggression and feeding, and may have skewed our dominance index. However, since body size is the determining factor in dominance relations among fish, a behavioural 
evaluation in the middle or at the end of an experiment may not have shown if the behaviour was the cause or the consequence of the greater SGR. In summary, our data do not permit us to conclude that the lower SGR of the more consanguineous individuals was the direct consequence of lower initial competitive ability in terms of the behavioural parameters evaluated in this study. Thus, other factors (ie the assimilation efficiency) may have determined the lower SGR: a hypothesis that remains to be evaluated in future research. In any case, as size differences increase, a synergic effect associated with territorial dominance would magnify the deleterious effects of inbreeding in competitive environments.

\section{Environmental dependence of ID: masking and magnifying effects}

In this study, we have shown that a change in social dominance relations may reverse or mask the deleterious effect of inbreeding in small groups of fish $(N=20)$. In order to do this, we manipulated the size relations between groups of fish with different levels of inbreeding, forcing the inbred fish to be dominant over the less inbred fish. Under these circumstances, the evidence of social dependence of ID was very consistent in relation to the dominant fish. In the two experiments carried out, the dominant fish expressed the same specific growth rate, independently of the level of inbreeding. The analysis is more complex, however, when evaluating the effect of inbreeding on subordinate individuals, since in one experiment both groups of subordinate fish (MI and LI) expressed the same SGR, but they did not in another experiment. This occurred in spite of the fact that the subordinate group (LI) of family 198 not only expressed a greater SGR than the subordinate fishes MI, but also a higher SGR than both MI and LI dominant groups. These data are consistent with differences in the expression of ID at the family level which has been described for other taxa (Holtsford, 1996), but may also suggest a compensatory effect as shown in the growth of the small fish.

We found at least two areas of evidence of environmental dependence of ID under culture conditions. First, the magnitude of the ID for body weight and the increase of SGR from the alevin to the smolt stage when fish are mixed in common cages. This coincides with previous observations carried out on this same population (unpublished data), as well as with published data on rainbow trout (Su et al, 1996). These authors showed that the magnitude of the ID for body weight increased with age: $\mathrm{ID}=+1.73 \%,-0.85 \%$ and $-2.26 \%$ for each $10 \%$ increment in inbreeding at 168, 280 and 364 days, respectively. Second, the greatest genetic load was expressed under high-density conditions, principally in the case of viability. Both results are consistent with a magnifying effect on ID in harsh environmental conditions similar to that observed for other taxa (Latter and Mulley, 1995; Haag et al, 2002). In plants, Cheptou et al (2001) found that ID on survival was greatest when inbred plants compete with outbred plants. However, as reviewed by Keller and Waller (2002), ID is not universally higher in stressful environments, suggesting that generalisation should be avoided.

The present results have important implications for aquaculture, and especially current programs for genetic improvement of fishes. For instance, Chilean salmon culture is based on a high degree of artificial selection (using a BLUP strategy), which has had a tendency to increase the rate of inbreeding. Although various methods have been devised to reduce this tendency (Villanueva et al, 1996; Gallardo et al, 2004b), it may be that a change in rearing conditions (ie lower densities) could also limit the expression of ID. At present there is a consensus that lowering culture densities could be beneficial for fishes, since stress is reduced, although this may produce an increase in genetic load for subsequent generations (Crow, 1997).

\section{Acknowledgements}

We thank Jean Paul Lhorente and Carlos Soto at the Coho Salmon Genetic Improvement Program (IFOPCoyhaique) for their professional support. JAG thank the National Commission on Science and Technology (CONICYT-CHILE) and the Universidad de Chile for the Doctoral Scholarship and financial support granted for this research.

\section{References}

Abbott JC, Dill LM (1985). Patterns of aggressive attack in juvenile steelhead trout (Salmo gairdneri). Can J Fish Aquat Sci 42: 1702-1706.

Bijlsma R, Bundgaard J, Van Putten WF (1999). Environmental dependence of inbreeding depression and purging in Drosophila melanogaster. J Evol Biol 12: 1125-1137.

Cheptou P-O, Lepart J, Escarre J (2001). Inbreeding depression under intraspecific competition in a highly outcrossing population of Crepis sancta (Asteraceae): Evidence for frequency-dependent variation. Am J Bot 88: 1424-1429.

Craik JC, Harvey SM, Jakupsstovu SHI, Shearer WM (1987). Identification of farmed and artificially reared Atlantic salmon among catches of the wild salmon fishery of the Faroes. ICES Council Meeting, 6pp.

Crnokrak P, Roff DA (1999). Inbreeding depression in the wild. Heredity 83: 260-270.

Crow JF (1997). The high spontaneous mutation rate: is it a health risk? Proc Natl Acad Sci USA 94: 8380-8386.

Cut CJ, Metcalfe NB, Taylor AC (2002). Fish may fight rather than feed in a novel environment: metabolic rate and feeding motivation in juvenile Atlantic salmon. J Fish Biol 61: 15401548.

Dahlgaard J, Hoffmann AA (2000). Stress resistance and environmental dependency of inbreeding depression in Drosophila melanogaster. Consv Biol 14: 1187-1192.

Dahlgaard J, Loeschke V (1997). Effects of inbreeding in three life stages of Drosophila buzzatii after embryos were exposed to a high temperature stress. Heredity 78: 410-416.

Damsgård B, Arnesen AM, Baardvik BM, Jobling M (1997). State dependent fish adquisition among two strains of hatchery-reared Artic charr. J Fish Biol 50: 859-869.

DeRose MA, Roff DA (1999). A comparison of inbreeding depression in life-history and morphological traits in animals. Evolution 53: 1288-1293.

Ejike C, Schreck CB (1980). Stress and social hierarchy rank in Coho salmon. Trans Am Fish Soc 109: 423-426.

Falconer DS, Mackay TFC (1996). Introduction to Quantitative Genetics, 4th edn. Logman Group: New York.

Fausch KD (1984). Profitable stream positions for salmonoids: relating specific growth rate to net energy gain. Can J Zool 62: $441-451$.

Gallardo JA, García X, Lhorente JP, Neira R (2004a). Inbreeding and inbreeding depression of female reproductive traits in 
two populations of Coho salmon selected using BLUP predictors of breeding values. Aquaculture 234: 111-122.

Gallardo JA, Lhorente JP, García X, Neira R (2004b). Effects of nonrandom mating schemes to delay the inbreeding accumulation in cultured populations of Coho salmon (Oncorhynchus kisutch). Can J Fish Aquat Sci 61: $547-553$.

Griffiths SW, Armstrong JD (2002). Kin-based territory overlap and food sharing among Atlantic salmon juveniles. J Anim Ecol 71: 480-486.

Haag CR, Hottinger JW, Riek M, Ebert D (2002). Strong inbreeding depression in a Daphnia metapopulation. Evolution 56: 518-526.

Hauser TP, Loeschcke V (1996). Drought stress and inbreeding depression in Lychnis flos-cuculi (Caryophyllaceae). Evolution 50: 1119-1126.

Healy BD, Lonzarich DG (2000). Microhabitat use and behavior of overwintering juvenile Coho salmon in a Lake Superior tributary. Trans Am Fish Soc 129: 866-872.

Hedrick PW, Kalinowski ST (2000). Inbreeding depression in conservation biology. Ann Rev Ecol Syst 31: 139-162.

Holtby LB, Swain DP, Allan GM (1993). Mirror-elicited agonistic behaviour and body morphology as predictors of dominance status in juvenile Coho salmon (Oncorhynchus kisutch). Can J Fish Aquat Sci 50: 676-684.

Holtsford T (1996). Variation in inbreeding depression among families and populations of Clarkia tembloriensis (Onagraceae). Heredity 76: 83-91

Huntingford FA, Metcalfe NB, Thorpe JE, Graham WD, Adams CE (1990). Social dominance and body size in Atlantic salmon parr, Salmo salar L. J Fish Biol 36: 877-881.

Keller LF, Waller DM (2002). Inbreeding effects in wild populations. TREE 17: 230-241.

Kelly WH (1967). Marking freshwater and marine fish by injected dyes. Trans Am Fish Soc 96: 163-175.

Kinghorn B, Kinghorn S (1999). PEDIGREE VIEWER Program Version 4.9. University of New England. Australia.

Lahti K, Laurila A, Enberg K, Piironen J (2001). Variation in aggressive behaviour and growth rate between populations and migratory forms in the brown trout, Salmo trutta. Anim Behav 62: 935-944.

Latter B, Mulley JC (1995). Genetic adaptation to captivity and inbreeding depression in small laboratory populations of Drosophila melanogaster. Genetics 139: 255-266.

Lynch M, Walsh B (1998). Genetics and Analysis of Quantitative Traits. Sinauer Associates: Sunderland, MA.

Martinez V, Neira R, Gall GAE (1999). Estimation of genetic parameters from pedigreed population. Lessons from analysis of alevin weight in coho salmon. Aquaculture 180: 223-236.

Meagher S, Penn D, Potts WK (2000). Male-male competition magnifies inbreeding depression in wild house mice. Proc Natl Acad Sci USA 97: 3324-3329.

Metcalfe NB, Huntingford FA, Graham WD, Thorpe JE (1989). Early social status and the development of life history strategies in Atlantic salmon. Proc Roy Soc London B 236: $7-19$.
Morton NE, Crow JF, Muller HJ (1956). An estimate of the mutational damage in man on data from consanguineous matings. Proc Natl Acad Sci USA 42: 855-863.

Neira R, Lhorente JP, Araneda C, Diaz NF, Bustos E, Alert A (2004). Studies on carcass quality traits in two populations of Coho salmon (Oncorhynchus kisutch): phenotypic and genetic parameters. Aquaculture 241: 117-241.

Nicieza AG, Metcalfe NB (1999). Costs of rapid growth: the risk of aggression is higher for fast-growing salmon. Func Ecol 13: $793-800$

Nevison CM, Barnard CJ, Beynon RJ, Hurst JL (2000). The consequences of inbreeding for recognising competitors. Proc Roy Soc London B 267: 687-694.

Pante MJ, Gjerde B, McMillan I (2001). Effects of inbreeding on body weight at harvest in rainbow trout, Oncorhynchus mykiss. Aquaculture 192: 201-211.

Quinn TP, Peterson NP (1996). The influence of habitat complexity and fish size on over-winter survival and growth of individually-marked juvenile coho salmon (Oncorhynchus kisutch) in Big Beef Creek, Washington. Can J Fish Aquat Sci 53: $1555-1564$.

Ryer CH, Olla BL (1996). Growth depensation and aggression in laboratory reared Coho salmon: the effect of food distribution and ration size. J Fish Biol 48: 686-694.

SAS Institute Inc (1996). SAS User's Guide: Statistics Windows Version 6.03. edition.

Sloman K, Metcalfe NB, Taylor AC, Gilmour KM (2001). Plasma cortisol concentration before and after stress in rainbow trout and brown trout. Physiol Biochem Zool 74: 383-389.

Sloman KA, Gilmour KM, Taylor AC, Metcalfe NB (2000). Physiological effects of dominance hierarchies within groups of brown trout, Salmo trutta, held under simulated natural conditions. Fish Physiol Biochem 22: 11-20.

Su GS, Liljedahl L-E, Gall GAE (1996). Effects of inbreeding on growth and reproductive traits in rainbow trout (Oncorhynchus mykiss). Aquaculture 142: 139-148.

Thorpe JE, Talbot C, Miles MS, Rawlings C, Keay DS (1990). Food consumption in 24 hours by Atlantic salmon (Salmo salar L. in a sea Cage. Aquaculture 90: 41-47.

Tiira K, Laurila A, Peuhkuri N, Piironen J, Ranta E, Primmer CR (2003). Aggressiveness is associated with genetic diversity in landlocked salmon (Salmo salar). Mol Ecol 12: 2399-2407.

Turnbull JF, Adams CE, Richards RH, Robertson DA (1998). Attack site and resultant damage during aggressive encounters in Atlantic Salmon (Salmo salar L. parr.). Aquaculture 159: 345-353.

Wang SZ, Hard J, Utter F (2001). Salmonid inbreeding: a review. Rev Fish Biol Fisher 11: 301-319.

Villanueva B, Woollians JA, Gjerde B (1996). Optimum designs for breeding programmes under mass selection with an application in fish breeding. Anim Sci 63: 563-576.

Vøllestad 1A, Quinn TP (2003). Trade-off between growth rate and aggression in juvenile coho salmon, Oncorhynchus kisutch. Anim Behav 66: 561-568.

Valdimarsson SK, Metcalfe NB (2001). Is the level of aggression and dispersion in territorial fish dependent on light intensity? Anim Behav 61: 1143-1149. 